\title{
LA REHABILITACIÓN ARQUITECTÓNICA PLANIFICADA
}

CLAUDIA TORRES

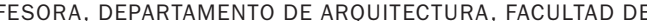

ARQUITECTURA Y URBANISMO, UNIVERSIDAD DE CHILE, SANTIAGO, CHILE
FIG 1A A FIG 1E

Vivienda minera en Lota, Región de Bio Bio, Chile. Pabellones rehabilitados bajo el Plan de Reconstrucción Patrimonial. Fotografías de Catalina Paredes, 2014.

\section{LA SOCIEDAD EN LA CONSERVACIÓN DEL PATRIMONIO}

$\mathbf{P}$

ara la conservación del patrimonio arquitectónico-entendido como una manifestación tangible de la expresión cultural de una sociedad cuyo valor trasciende como bien heredable a futuras generaciones- se buscan métodos de valoración, recuperación, actualización, mantenimiento y difusión de dichos valores en base a modelos que sean sostenibles a largo plazo y que puedan asegurar su permanencia futura. En este sentido, las visiones contemporáneas de conservación del patrimonio (declaradas en cartas internacionales como la Carta de Cracovia 20oo) definen líneas de integración entre la arquitectura, como manifestación construida de las formas y condiciones de vida de una sociedad durante un determinado periodo, y las expresiones culturales de sus habitantes, implicando la preservación de obras, lugares y zonas de diversa valoración cultural y patrimonial.
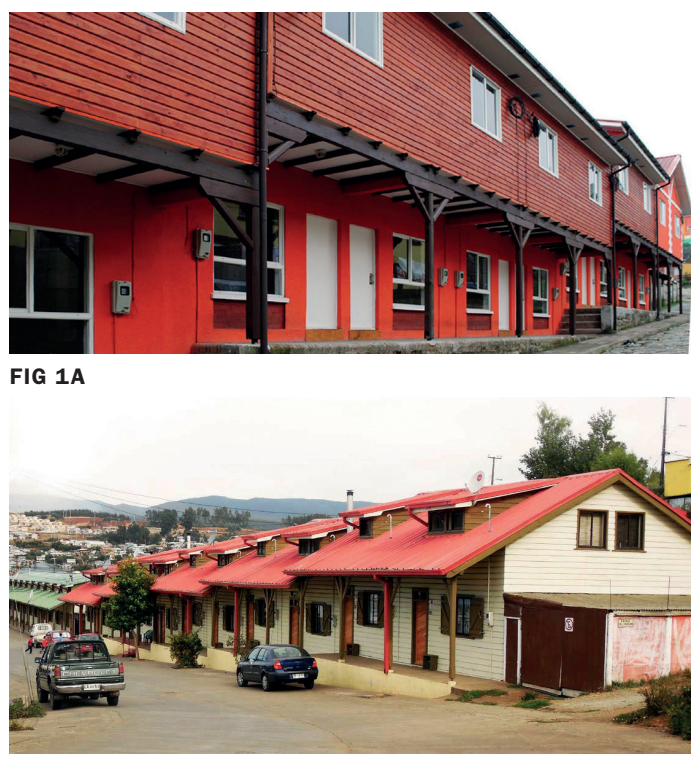

FIG 1B

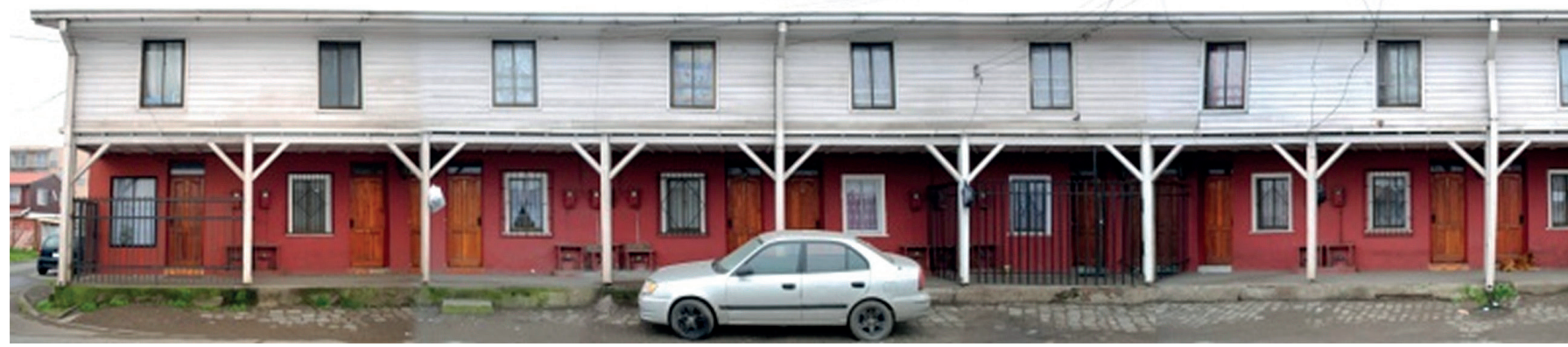

En este aspecto hemos avanzado significativamente en la última década, pasando de la protección y financiamiento para la conservación de obras singulares y monumentales (principalmente de carácter religioso), a la protección (con menos recursos) para conjuntos urbanos, industriales u obras de menor alcance, anónimas o constitutivas de un determinado paisaje patrimonial. Así es como por parte del Estado chileno se han desarrollado planes como el de "Puesta en valor del patrimonio" a cargo del MOP y con financiamiento del BID iniciado el año 2007, cuyo objetivo es la recuperación del patrimonio protegido bajo la Ley de Monumentos Nacionales, o el "Plan de reconstrucción patrimonial” a cargo del MINVU, iniciado el 2011 a partir del terremoto del 2010 y cuyo objetivo es la entrega de subvenciones para la recuperación de viviendas ubicadas en zonas patrimoniales (fig. 1).

No obstante, pareciera que aún nos falta asimilar el relevante rol que cumplen o debieran cumplir los diversos actores de nuestra sociedad en la
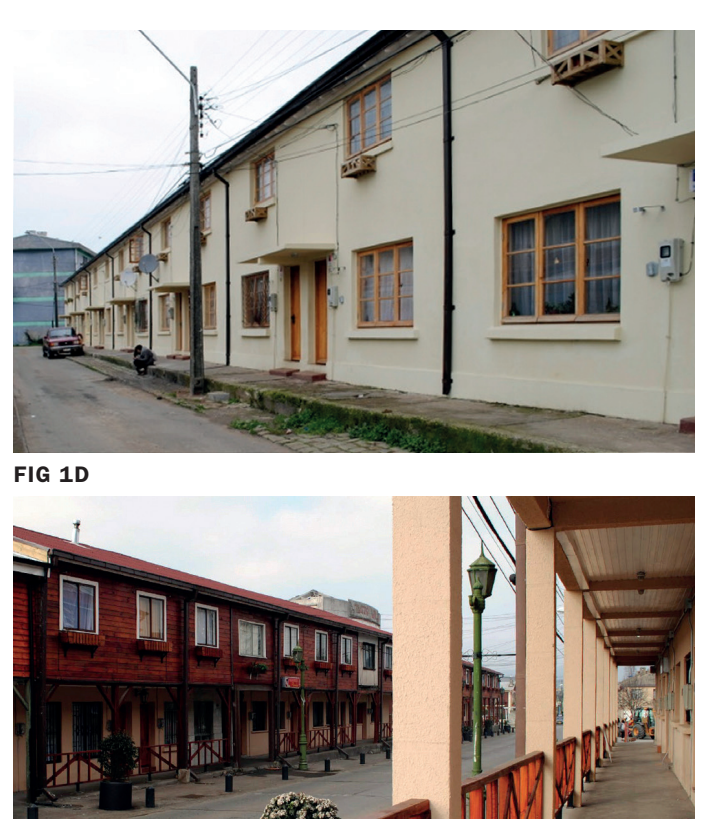

FIG 1E 
FIG 2A A FIG 2E

Viviendas en Vichuquén, Región del

Maule, Chile. En proceso de rehabilitación post-terremoto 2010, bajo el Plan de

Recuperación Patrimonial Zona Típica de Vichuquén.

Fotografías de la autora, 2012
Esta institución se ha encargado de finan- 3 ciar proyectos pertenecientes al "Plan de recuperación patrimonial" y a los del "Plan

de reparaciones mayores", posteriores al terremoto de 2010 en Chile.
La rehabilitación arquitectónica se constituyó en este caso como herramienta para revertir un proceso o política social de liberación de terrenos en centros urbanos con plusvalía, servicios y conectividad.

La rehabilitación de los barrios antiguos debe ser concebida y realizada, en la medida de lo posible, de forma que no modifique sustancialmente la composición social de los residentes y permitiendo que todos los estratos de la sociedad se beneficien de una operación financiada mediante fondos públicos (Consejo de Europa, 1975). Actualmente, en un contexto de creciente diversidad de proyectos que buscan la recuperación y conservación del patrimonio arquitectónico, la rehabilitación arquitectónica es entendida como el conjunto de acciones que permiten habilitar un lugar o una obra existente recuperando las funciones desempeñadas en ellos, o bien, integrándoles nuevos usos. Es decir, habilitar los espacios de modo que puedan reincorporarse funcionalmente a la sociedad, adaptándose a formas de vida contemporánea y que, mediante una nueva significación, se integren como parte de la memoria colectiva de los habitantes. "Rehabilitar implica mejorar el hecho de habitar, buscando el equilibrio entre los aspectos técnicos, la preservación de los valores patrimoniales y los criterios de equidad social, de eficiencia económica y de preservación del medio ambiente (los tres puntales de la sostenibilidad)" (RehabiMed, 2005, p. 13).

Por otra parte, no se puede desconocer que la mayoría de los proyectos de rehabilitación gestionados por entidades privadas buscan una rentabilidad económica de la inversión, buscando hacer sustentable tanto la preservación y mantenimiento de la obra, como las actividades que en ellas se desarrollan. En este punto se diferencian de los proyectos desarrollados con financiamiento público. Estos últimos, más bien buscan una rentabilidad social de la inversión, como sucede con los múltiples casos de rehabilitación de vivienda social en España o, a nivel nacional, con la rehabilitación de establecimientos educacionales llevados a cabo por el Ministerio de Educación ${ }^{3}$, los municipios y gobiernos regionales (figs. 3 y 4). Otro caso emblemático es la rehabilitación de la Población Obrera, en el cerro Cordillera de Valparaíso, desarrollado bajo un modelo mixto de gestión y financiamiento (Araya, 2009).

La predominancia de los factores de funcionalidad y la búsqueda de una posible rentabilidad económica del proyecto también son condiciones que diferencian los proyectos de rehabilitación con los de restauración. En estos últimos se busca recuperar las característica originales que dan valor a la obra arqui- tectónica como patrimonio cultural, muchas veces no importando el costo económico que implique la intervención, dado que sus valores históricos, artísticos o singulares así lo ameritan, dando prioridad a los factores de durabilidad: "La restauración arquitectónica es una intervención dirigida sobre un bien patrimonial, cuyo objetivo es la conservación de su autenticidad y su apropiación por la comunidad" (Conferencia Internacional sobre Conservación, 2000, p. 5). Sin embargo, hoy en día en este tipo de proyectos se comienza a entender que, una vez que se haya realizado el proyecto de restauración, es necesario desarrollar gestiones integrales que permitan asegurar una autonomía de recursos para el mantenimiento del inmueble y su entorno inmediato.

\section{LA OBSOLESCENCIA, LOS TERREMOTOS Y LA OBLIGACIÓN: VARIABLES QUE DAN ORIGEN A UN PROYECTO DE REHABILITACIÓN}

Comprendiendo que la rehabilitación es un proceso cuyo objetivo primordial es mejorar las condiciones de vida de los habitantes mediante las mejoras del medio construido, actualmente vemos que estos proyectos se desarrollan, primordialmente, en situaciones de áreas urbanas o edificaciones cuyos modelos espaciales y funcionales se encuentran obsoletos en relación a los modelos contemporáneos de desarrollo social. Algunos casos característicos de este tipo de proyectos, llevados a cabo usualmente en Europa son: los proceso de reconversión de áreas industriales obsoletas productivamente y cuyos valores y potenciales son reconocidos internacionalmente mediante la Carta de Nizhny Tagil (2003) sobre el patrimonio industrial; también, la reutilización de conjuntos religiosos, obsoletos por falta de vocaciones y altos costos de mantenimiento; las reformas urbanas y planes de revitalización en centros históricos degradados o abandonados; o la rehabilitación de viviendas tradicionales o conjuntos rurales deteriorados, sobre o subutilizados.

No obstante, en nuestro contexto nacional vemos que gran parte de las rehabilitaciones desarrolladas en el último tiempo tienen como objeto la recuperación de un patrimonio dañado por las acciones sísmicas. Es decir, existe una variable que, en un país sísmico como Chile, obliga al emprendimiento de gestiones específicas para la recuperación de estas edificaciones y que tiene relación con la vulnerabilidad constructivo-estructural de las obras y las lesiones de origen mecánico que las han afectado producto de los terremotos. Por lo tanto, la obsolescencia o el abandono funcional de una obra arquitectónica no son las únicas condiciones que originan un proyecto de rehabilitación.

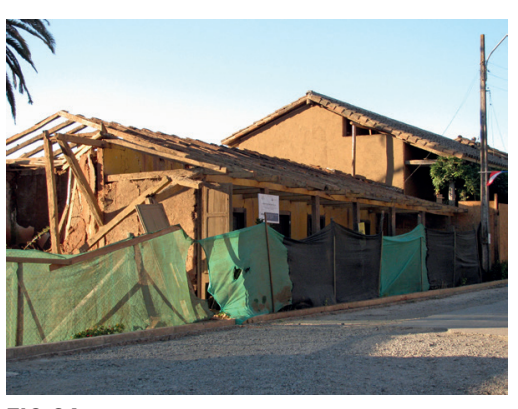

FIG 2A

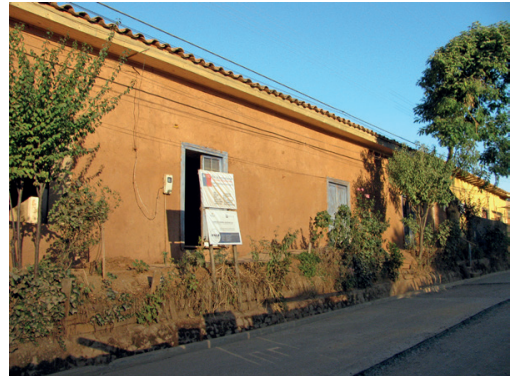

FIG 2B

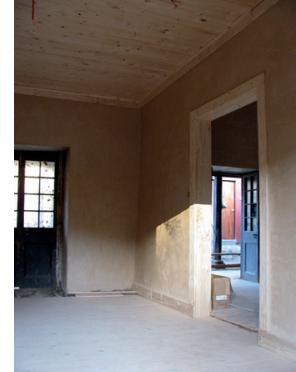

FIG 2C

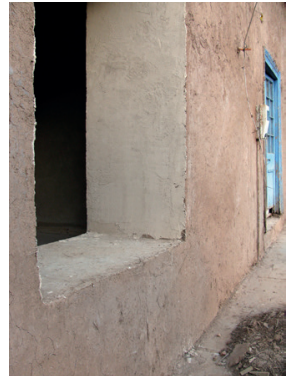

FIG 2D

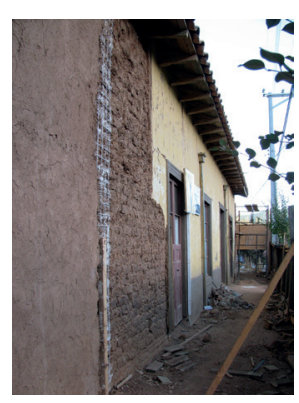

FIG 2E 

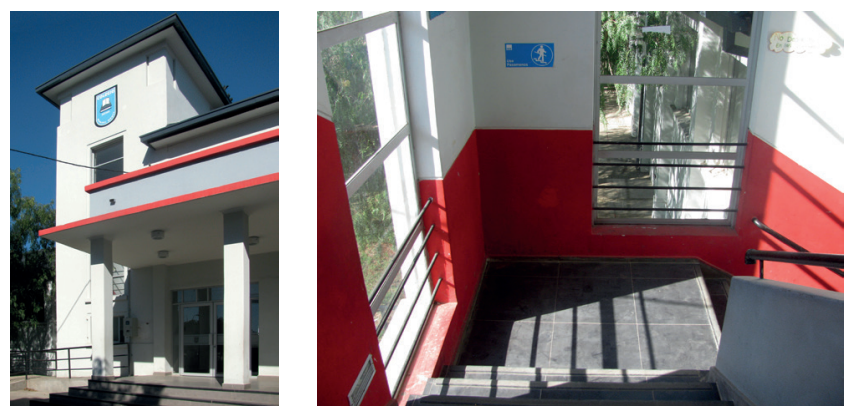

FIG 3B

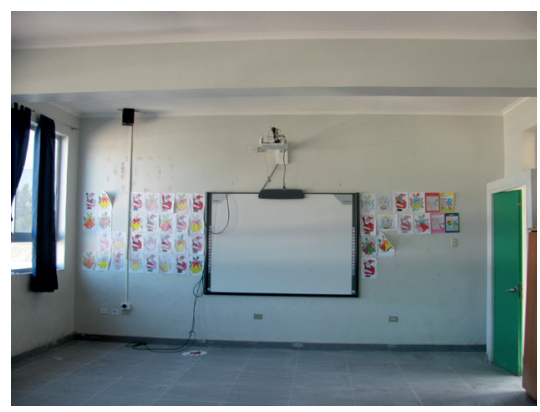

FIG 3C
FIG 3A FIG 3 C

Proyecto de Rehabilitación de la Escuela Manuel Montt de Retiro, Región del Maule, Chile. Arquitecto: Boris Mascaró. Fotografías de la autora, 2014

CUADRO 1

Variables que condicionan los proyectos de Rehabilitación Arquitectónica.
En Chile, los proyectos con algún tipo 4 de valoración y protección patrimonial quedan afectos a la OGUC al igual que un proyecto de obra nueva y además pueden estar afectos a regulaciones adicionales de algún plan seccional (si son ICH o se emplazan en una $\mathrm{ZCH}$ ), o a las recomendaciones de algún instructivo de zona típica si cuentan con protección bajo la Ley de Monumentos Nacionales.
Asimismo, como tercer aspecto de análisis, tenemos que las zonas e inmuebles que cuentan con algún tipo de protección legal de patrimonio, ya sea bajo la Ley de Monumentos (monumento histórico, zonas típicas) o bien bajo la protección de los 'Planes seccionales' de los respectivos Planes Reguladores Comunales PRC (inmuebles de conservación histórica ICH, o zonas de conservación histórica ZCH), están bajo un régimen de conservación obligada, ya que no es posible su demolición. Sin embargo, es factible realizar intervenciones de forma controlada, especialmente en los inmuebles cuya valoración patrimonial está definida a partir de las características tipológicas de su arquitectura. La rehabilitación se convierte en la opción viable de intervención, ya que es un modelo de intervención que puede garantizar la conservación de los valores primordiales de la obra arquitectónica (en cuanto a sí misma o en relación a su contexto) y a su vez incorporar preceptos contemporáneos de habitabilidad (fig. 5).

En general, en los proyectos de rehabilitación se emprenden actuaciones de intervención en los inmuebles o lugares respondiendo a variables específicas que condicionan el diseño del proyecto y que permiten hacer sostenible la conservación de los valores arquitectónicos, culturales e identitarios de la obra (ver cuadro 1). Las principales variables (además de las económicas, que en general condicionan la mayoría de las obras) son las condiciones constructivas de conservación de la obra preexistente, las condiciones de riesgo según su emplazamiento, los objetivos funcionales del proyecto y las regulaciones normativas. ${ }^{4}$

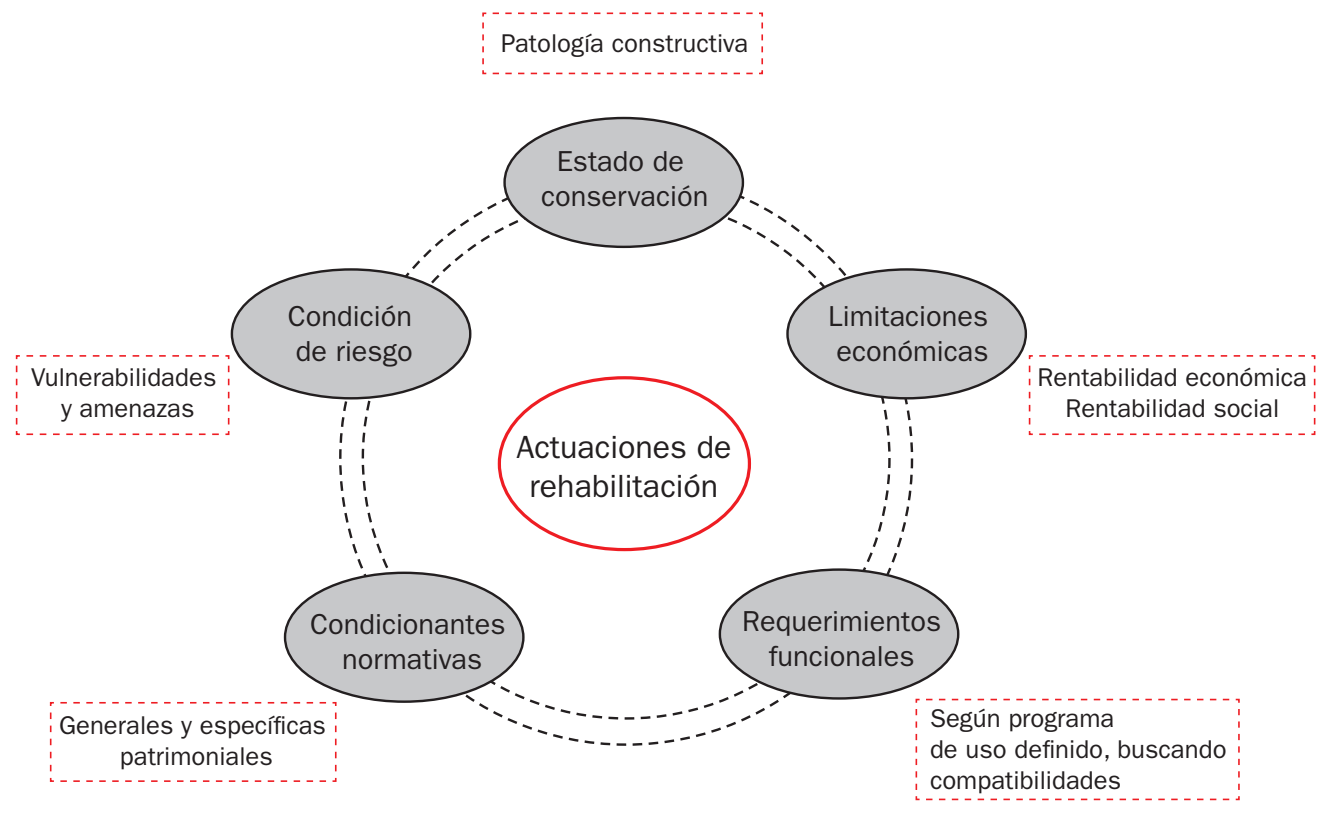

CUADRO 1

En relación a las normativas, salvo lineamientos de conservación volumétrica, en el contexto chileno no existen normas específicas para la intervención en el patrimonio arquitectónico como sí las hay en otros países, como por ejemplo en España, donde en el año 2013 se ha aprobado a nivel nacional la Ley 8/2013 de 'Rehabilitación, regeneración y renovación urbanas' como complemento a otras ya existentes en cada comunidad, tales como la Ordenanza Metropolitana de Rehabilitación vigente desde 1985 en Barcelona.

\section{TIPOS DE REHABILITACIÓN ARQUITECTÓNICA}

Teniendo como referencia el estudio de algunos planes, normativas y regulaciones españolas, así como también los proyectos de rehabilitación realizados en inmuebles tanto de España como de Chile, las actuaciones de rehabilitación en las edificaciones se pueden clasificar según el alcance, origen y tipo de obras realizadas en:

REHABILITACIONES INTEGRALES: son aquellas actuaciones que suponen una intervención sobre todos los elementos del edificio que lo requieran, ya sean estructurales, constructivos o distributivos, produciéndose o no una alteración significativa de los espacios originales y su volumetría, mejorando sus condiciones funcionales o adaptándose a nuevos usos. En general, este tipo de intervenciones son ejecutadas en inmuebles de un único propietario o en inmuebles de propiedad vertical, ya que esto facilita las gestiones y la rapidez en la toma de decisiones. Normalmente las rehabilitaciones se realizan cuando la edificación presenta un cuadro patológico grave que afecta a la mayoría de la edificación-sobre todo cuando la estructura se encuentra comprometida-; cuando existe un interés inmobiliario por llevar a cabo una operación de densificación, cuando se quiere hacer una reconversión funcional de la edificación, o bien cuando existe una política pública interesada en rescatar ciertas edificaciones a modo de intervención ejemplar.

REHABILITACIONES PARCIALES: son consideradas como aquellas intervenciones que no operan sobre la totalidad de los inmuebles. Es decir, obras de reparaciones, consolidaciones, reconstrucción de elementos comunes (estructura, cubierta, fachada, accesibilidad o espacios comunes), ampliaciones, como también transformaciones espaciales que afectan un nivel o piso en particular. En general se desarrollan en edificaciones con régimen de propiedad horizontal cuando se requiere mejorar la imagen del edificio o habilitar instalaciones necesarias en espacios comunes. Estas intervenciones habitualmente son más lentas en las labores previas de coordinación entre todos los propietarios involucrados y los tiempos de gestión del proyecto se prolongan por las dificultades en la toma de decisiones. Sin embargo, normalmente cuentan con facilidades en el acceso a programas de ayudas económicas o subvenciones. Normalmente se ejecutan en inmuebles con daños leves que no requieren una intervención en la totalidad de la estructura, en caso de ejecución de modificaciones estéticas para actualizar la imagen de la edificación o cuando se hacen cambios de usos de menor impacto, como por ejemplo el cambio de un uso residencial a un uso hotelero. 


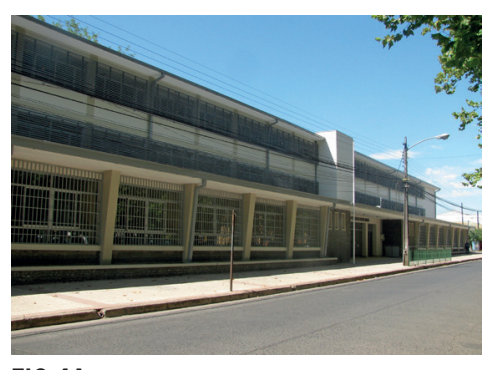

FIG 4A

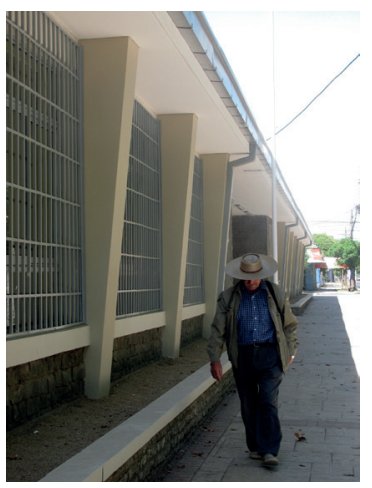

FIG 4B

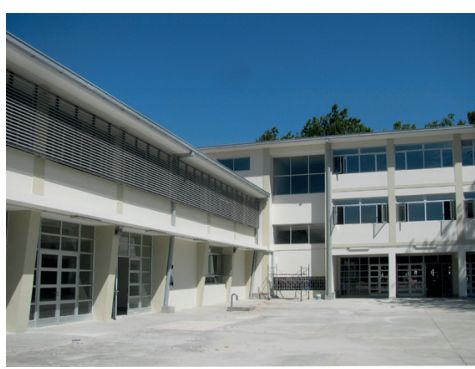

FIG 4C

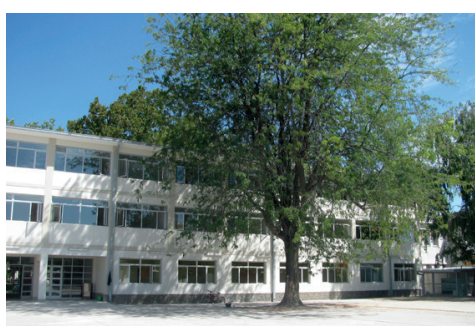

FIG 4D

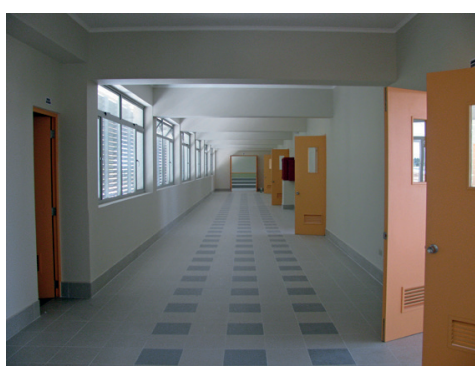

FIG 4E

En el Anexo A de la norma chilena NCh. 5 433 de 1996 se plantean de modo informativo los criterios y procedimientos para la evaluación y recuperación de las estructuras dañadas por sismo.
REHABILITACIONES INTERIORES: son aquellas intervenciones privativas de un recinto interior que permiten asumir las condiciones de espacio habitable con redistribuciones que mejoran las condiciones funcionales del uso actual o nuevo, normalmente incorporando nuevas redes e instalaciones sin alterar los sistemas estructurales de la obra ni sus paramentos externos. Generalmente este tipo de actuación se realiza a partir de un cambio de uso que requiere la adaptación y actualización de los espacios por nuevos requerimientos normativos de habitabilidad y seguridad. También es frecuente este tipo de rehabilitaciones cuando se realizan subdivisiones interiores en grandes edificaciones.

\section{ACTUACIONES DE REHABILITACIÓN}

En base a los casos de rehabilitación estudiados, tanto en inmuebles residenciales como institucionales, es posible establecer que en ellos se busca adaptar la obra existente a formas contemporáneas de habitabilidad según el uso propuesto. Estos proyectos se llevan a cabo con actuaciones que, por una parte, intervienen las condiciones espaciales de la obra y, por otra, intervienen los sistemas constructivo-estructurales del inmueble, además de intentar actualizar los sistemas de instalaciones, normalmente obsoletos (ver cuadro 2). Al proponer intervenciones que no desvirtúen el sentido original de la obra en cada uno de estos ámbitos de actuación, los proyectos presentan desafíos significativos para cumplir las normativas que los regulan. Estas dificultades se encuentran directamente relacionadas con los aspectos morfológicos y tipológicos de las edificaciones existentes y su parcelación.

De modo más específico encontramos que en estos proyectos se modifica la distribución de los espacios interiores, transformando elementos secundarios para lograr solucionar las usuales dificultades en las condiciones de iluminación y ventilación $y$, a su vez, ordenar las áreas de circulación, mejorando las condiciones de accesibilidad al inmueble y a los recintos interiores. De manera preponderante, factores como la cantidad y el ancho de las fachadas o la existencia de patios de luz influyen o restringen las posibilidades de diseño, siendo muchas veces determinantes en los posibles usos del inmueble y en las subdivisiones espaciales planteadas para rentabilizar los espacios residuales que quedan sin ventilación ni iluminación natural.

Por otra parte, se realizan intervenciones que buscan aumentar los niveles de seguridad de la obra actuando en el sistema constructivo-estructural del inmueble mediante su consolidación, reparación o refuerzo, de modo que se asegure tanto su estabilidad y resistencia sísmica ${ }^{5}$ como los niveles de protección contra incendios. Además, en nuestro país se aprovecha este tipo de intervenciones para incorporar elementos de protección en las zonas vulnerables frente a posibles robos.

En general, vemos que se busca mejorar la eficiencia energética del inmueble sólo cuando es un requerimiento específico del proyecto, ya sea interviniendo sobre la envolvente o bien incorporando sistemas pasivos de eficiencia energética. Esto ya es parte de las regulaciones y exigencias en proyectos europeos, no así en nuestro país. A pesar de ello, en algunos casos se realizan intervenciones que buscan mejorar las condiciones de confort interno mediante elementos de aislación tanto acústicos como térmicos.

Por último, en todos los proyectos se eliminan los sistemas de instalaciones existentes ya que normalmente no cumplen las regulaciones actuales, por eso se renuevan incorporando nuevos sistemas de redes e instalaciones de mayor complejidad en casos de requerimientos específicos del proyecto.

Dado que las terminaciones de los proyectos tienen relación con la imagen que se espera proyectar de la obra, cuando en el inmueble existen algunos elementos de valor artístico o significativo, estos suelen conservarse dando cuenta de la antigüedad o condiciones tradicionales de la obra. No obstante, en general los proyectos buscan incorporar elementos nuevos que entreguen una visión de espacios renovados bajo un lenguaje de arquitectura contemporánea.

En casos de proyectos en que se busca aumentar la superficie construida del inmueble para hacerlo rentable, ya sea con ampliaciones de volúmenes en las zonas no construidas del terreno o bien mediante remontas, las restricciones volumétricas quedan sujetas a las regulaciones de las normativas locales o a los criterios compositivos del arquitecto responsable del proyecto, quien muchas veces es presionado para lograr mayor rentabilidad con el proyecto, en desmedro de los valores patrimoniales del inmueble.

\section{REFLEXIONES GENERALES}

La rehabilitación como proceso de recuperación y conservación del patrimonio arquitectónico desde una visión dinámica de sus valores, entendiendo que "los elementos individuales de este patrimonio son portadores de muchos valores, los cuales pueden cambiar en el tiempo" (Conferencia Internacional sobre Conservación, 200o, p. 1), permite adaptar la obra existente a nuevos requerimientos de uso y con ello extender la vida útil del inmueble, incorporándolo a un nuevo ciclo de funcionalidad dentro del desarrollo social y cultural de una comunidad.

Podemos ver que los proyectos de rehabilitación como modelos de preservación del patrimonio están ampliamente desarrollados en países europeos y hoy recién comienzan a gestarse en nuestras ciudades con las dificultades propias de un proceso germinal poco regulado y bajo un modelo que, buscando cierta rentabilidad, se opone muchas veces a los intereses ciudadanos. Es por esto que se deben estudiar en profundidad los fenómenos locales y las vulnerabilidades sociales y arquitectónicas de estos inmuebles y su entorno, así como también sus posibilidades de recuperación de manera sostenible en el tiempo.

Los planes que se han desarrollado en Europa en base a una rehabilitación planificada abocada a salvaguardar los centros históricos de las fuertes presiones inmobiliarias y las diversas estrategias asumidas para revertir estos procesos, se convierten en ejemplos para ciudades que se inician en estos procesos de forma tardía, teniendo, en nuestro caso, la ventaja de generar nuevas estrategias en base a los aciertos y errores cometidos en ellos. 


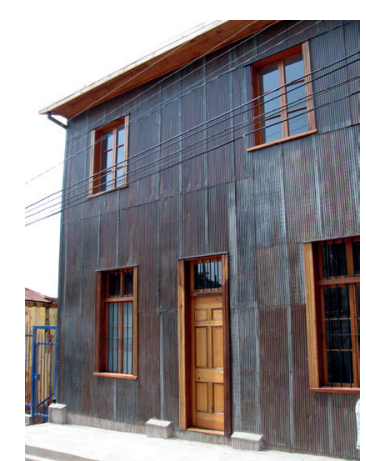

FIG 5A

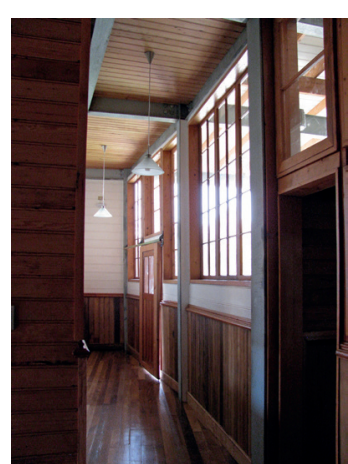

FIG 5B

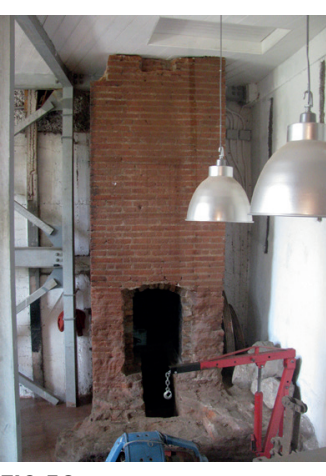

FIG 5C

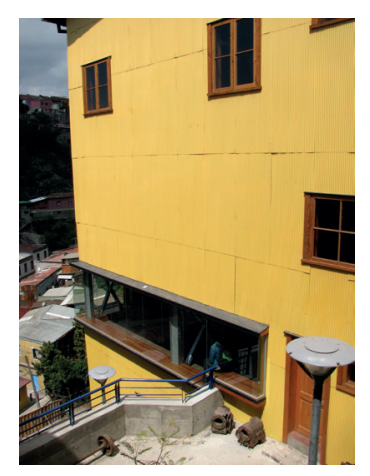

FIG 5D

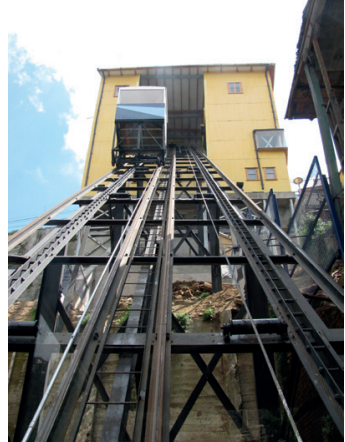

FIG 5E

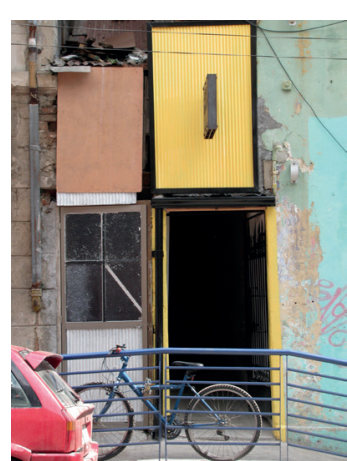

FIG 5F
FIG 5A A FIG 5F

Rehabilitación del Ascensor San Agustín en cerro Cordillera, Valparaíso, Chile. Fotografías de la autora, 2012

\section{CUADRO 2}

Ámbitos de actuación en los proyectos de Rehabilitación Arquitectónica.
Frente a la falta de una legislación específica para los proyectos de rehabilitación a nivel nacional, hemos visto que no existe en los instrumentos reguladores una clasificación de los alcances o tipo de actuaciones desarrolladas en estos proyectos, así como tampoco existe un planteo o visión de los procesos de rehabilitación como política pública por parte de las instituciones que participan en estos procesos. Por lo tanto, mediante estos análisis se ha podido encaminar una clasificación general aplicable a cualquier proyecto o inmueble en proceso de rehabilitación.

Desde los aspectos de la rehabilitación arquitectónica como mercado naciente, es importante señalar que se debe avanzar en contar con profesionales y mano de obra especializada, así como también es necesario mejorar las capacidades técnicas que permitan tener un mayor conocimiento de las características y condiciones constructivas de las edificaciones preexistentes, ya que de esto dependerán los tipos de intervenciones diseñadas, los costos y tiempos de ejecución de los proyectos.

Evidentemente las condiciones geográficas de nuestro territorio nacional, principalmente la condición sísmica, nos hacen reaccionar de modo eventual creando y gestionando programas que financien algunos niveles de intervención de rehabilitación, intentando la recuperación o mantenimiento de nuestro patrimonio arquitectónico. Sin embargo, si estos programas fuesen planificados con financiamiento a largo plazo, disminuiríamos las vulnerabilidades de estas obras. Así comienza a hacer necesario el diseño de una política de rehabilitación arquitectónica planificada con modelos de gestión de recursos públicos y privados, de modo que se pueda lograr con esto una rentabilidad económica, pero principalmente social. ARQ

\section{REFERENCIAS BIBLIOGRÁFICAS}

ALLAIN, Jean. "Une Politique en Marche". En: DUPORT, Jean Pierre. 40 ans de Réhabilitation de l'habitat en France. París, Economica, 1989. p. 61-70.

ARAYA, Raúl; CASTILLO, María José y PRADO, Francisco. "Rehabilitación Unión Obrera: Cerro Cordillera". ARQ, (73): 3639, diciembre de 2009.

CONFERENCIA INTERNACIONAL SOBRE CONSERVACIÓN. Carta de Cracovia 2000. Principios para la conservación y restauración del patrimonio construido [en línea]. Cracovia, 2000 [fecha de consulta: 13 de octubre de 2014]. Disponible en: <http://ipce. mcu.es/pdfs/2000_Carta_Cracovia.pdf>

CONSEJO DE EUROPA. Declaración de Ámsterdam, carta europea del patrimonio arquitectónico [en línea]. Holanda, 1975 [fecha de consulta: 13 de octubre de 2014]. Disponible en: <http://www. icomoscr.org/doc/teoria/VARIOS.1975.declaracion.amsterdam. patrimonio.arquitectonico.europeo.pdf $>$ REHABIMED. Método RehabiMed. Arquitectura tradicional mediterránea. I Rehabilitación, ciudad y territorio [en línea]. Barcelona, 2005 [fecha de consulta: 23 de octubre de 2014]. Disponible en: <http://www.rehabimed.net/Publicacions/ Metode_Rehabimed/I.Rehabilitacio_Ciutat_i_Territori/ES/1a\%20 parte.pdf>

\section{BIBLIOGRAFÍA}

ABELLA, Marti. Ciutat Vella, Rehabilitación Integral en el Centro Histórico de Barcelona [en línea]. Barcelona, 1996 [fecha de consulta: 23 de octubre de 2014]. Disponible en: <http:// habitat.aq.upm.es/dubai/96/bp259.html>

RESOLUCIÓN N 152. Aprueba formato de bases tipo administrativas, técnicas y anexos para "Planes de Reparaciones Mayores" y convenios tipo para Establecimientos Educacionales Municipales y Particulares Subvencionados afectados por el terremoto de 27 de febrero del año 2010. MINEDUC, Santiago, Chile, 21 de junio de 2011. Disponible en: <http://www. contraloria.cl/NewPortal2/portal2/ShowProperty/BEA\%20 Repository/Portal/Destacados/Bases_Aprobadas/Juridica/ Res_152.PDF>

LEY N 8/2013, 26 de Junio, de rehabilitación, regeneración y renovación urbanas. Boletín Oficial del Estado, España, 27 de junio de 2013. Disponible en: <http://www.minhap.gob.es/ Documentacion/Publico/NormativaDoctrina/Tributaria/IRPF/ Ley_8-2013.pdf>

MINEDUC. Nuevos espacios educativos. 2010-2013 [en línea]. Santiago, 2014 [fecha de consulta: 11 de octubre de 2014]. Disponible en: <http://www.mineduc.cl/usuarios/mineduc/doc/ NuevosEspaciosEducativos.pdf>

TICCIH. Carta de Nizhny Tagil sobre el patrimonio industrial [en línea]. Moscú, 2003 [fecha de consulta: 17 de octubre de 2014]. Disponible en: <http://international.icomos.org/18thapril/2006/ nizhny-tagil-charter-sp.pdf>

TORRES, Claudia. La rehabilitación de viviendas a partir de Ios Planes Especiales de Reforma Interior (PERIS), en el centro histórico de Barcelona. 1990-2004. Estudio arquitectónico, constructivo-estructural de las propuestas de intervención, en Ios barrios de La Barceloneta y El Raval. Tesis (Doctora en arquitectura). Barcelona, Departamento de Construcciones Arquitectónicas I, ETSAB, UPC, 2012.

CLAUDIA TORRES | Arquitecta, Universidad de Valparaíso, 1998; Doctora en Arquitectura, Universidad Politécnica de Cataluña, 2012. Actualmente se desempeña como docente en la Facultad de Arquitectura y Urbanismo de la Universidad de Chile. Tanto su tesis doctoral como los cursos que imparte están vinculados a la investigación y el estudio de los aspectos tecnológicos en la conservación del patrimonio. Además de su labor docente participa en proyectos de restauración y rehabilitación patrimonial, principalmente en proyectos del programa "Puesta en valor del patrimonio" del Ministerio de Obras Públicas de Chile. Varios de sus estudios docentes y trabajos profesionales se encuentran publicados en congresos relacionados al diagnóstico y rehabilitación del patrimonio. 